\title{
The OLYMPUS Internal Hydrogen Target
}

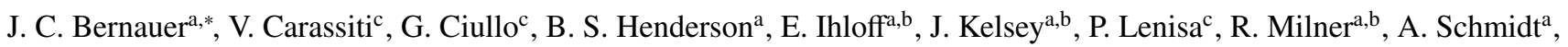
M. Statera ${ }^{\mathrm{c}}$

${ }^{a}$ Massachusetts Institute of Technology, Laboratory for Nuclear Science, Cambridge, MA 02139, USA

${ }^{b}$ MIT-Bates Linear Accelerator Center, Middleton, MA 01949, USA

${ }^{c}$ Istituto Nazionale di Fisica Nucleare and Università, 44100, Ferrara, Italy

\begin{abstract}
An internal hydrogen target system was developed for the OLYMPUS experiment at DESY, in Hamburg, Germany. The target consisted of a long, thin-walled, tubular cell within an aluminum scattering chamber. Hydrogen entered at the center of the cell and exited through the ends, where it was removed from the beamline by a multistage pumping system. A cryogenic coldhead cooled the target cell to counteract heating from the beam and increase the density of hydrogen in the target. A fixed collimator protected the cell from synchrotron radiation and the beam halo. A series of wakefield suppressors reduced heating from beam wakefields. The target system was installed within the DORIS storage ring and was successfully operated during the course of the OLYMPUS experiment in 2012. Information on the design, fabrication, and performance of the target system is reported.
\end{abstract}

N Keywords:

internal hydrogen target, OLYMPUS, wakefield suppression, vacuum system PACS: 29.25.Pj, 25.30.Bf, 07.05.Fb, 07.20.MC, 07.30.Cy, 07.30.Kf, 07.05.Dz

\section{Motivation}

The OLYMPUS experiment [1] at DESY, in Hamburg, Germany, was designed to measure the cross section ratio of electron-proton to positron-proton elastic scattering over the range of negative four-momentum transfer from 0.4 to $2.2(\mathrm{GeV} / c)^{2}$ at a fixed beam energy of $2.01 \mathrm{GeV}$. A contribution to the cross section from hard two-photon exchange would cause this ratio to deviate from unity. Such contributions are believed to at least partially resolve the discrepancy in the proton form factor ratio $G_{E} / G_{M}$ measured in Rosenbluth separation and polarization experiments [2]. There is no theoretical consensus on the size of the two-photon contribution [3], and a direct experimental measurement is needed. The design goal for OLYMPUS was to measure this ratio with less than $1 \%$ uncertainty.

The OLYMPUS detector was installed at the DORIS storage ring at DESY, the layout of which is shown in Fig. 1 DORIS was selected because it could circulate both electron and positron beams with multi-GeV energies and currents up to $150 \mathrm{~mA}$. OLYMPUS aimed to measure the elastic cross section ratio by directing the DORIS beam through an internal hydrogen gas target, switching beam species about once per day. The scattered lepton and the recoiling proton were detected in coincidence. For its detector systems, OLYMPUS incorporated several components originally used in the Bates Large Acceptance Spectrometer Toroid (BLAST) experiment [4]. The differences in physics goals and beam environments between OLYMPUS

\footnotetext{
${ }^{*}$ Corresponding author

Email address: bernauer@mit.edu (J. C. Bernauer)
}

and BLAST, however, required the design of a new unpolarized hydrogen target system. The target system faced three principal technical challenges:

1. In order for OLYMPUS to attain a sufficient luminosity, a target thickness of $3 \times 10^{15}$ atoms $/ \mathrm{cm}^{2}$ was required.

2. The high beam current made it infeasible to have the beam pass through target windows. Therefore, a multistage pumping system was required to avoid spoiling the DORIS beamline vacuum with hydrogen from the target.

3. The bunches in DORIS could carry as much as $30 \mathrm{nC}$ each. With such a high charge per bunch, any conducting structures close to the beam were susceptible to intense wakefield heating. To reduce the heat load, extensive wakefield suppression was needed.

To meet these technical demands, the OLYMPUS target was designed as a windowless gaseous target, internal to the DORIS ring. The DORIS beam passed through an elliptical tubular target cell into which $\mathrm{H}_{2}$ gas was flowed. The gas was fed into the center of the cell and was removed by a three-stage differential pumping system as it flowed out the ends of the cell. A fixed tungsten collimator was positioned in front of the cell to protect it from synchrotron radiation and the beam halo. The cell, collimator, and beam pipe were all linked by conducting transitions to suppress wakefields. The cell was cooled by a cryogenic coldhead to increase the target density and to remove heat produced by wakefields.

When not providing a beam for OLYMPUS, the DORIS storage ring served as a synchrotron light source. During OLYMPUS data taking, DORIS circulated 40 to $65 \mathrm{~mA}$ beams in ten 


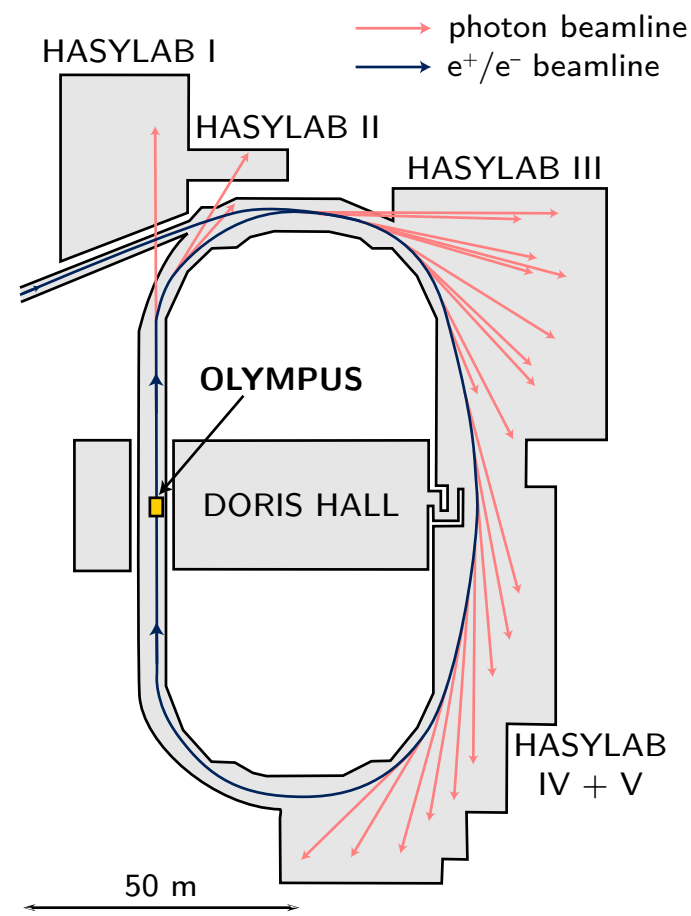

Figure 1: The layout of the DORIS $e^{+} / e^{-}$storage ring at DESY, showing the position of the OLYMPUS detector and the synchrotron light experiment stations.

bunches at an energy of $2.01 \mathrm{GeV}$. In light source mode, DORIS circulated $140 \mathrm{~mA}$ positron beams in five bunches at an energy of $4.45 \mathrm{GeV}$. Due to the impracticality of removing the target system, the OLYMPUS target was designed to tolerate the significantly more taxing conditions of light source operation.

\section{Design}

\subsection{Scattering Chamber}

The target cell, collimator, and wakefield suppressors were housed within an aluminum scattering chamber, which is shown in Fig. 2a. The DORIS beam was directed through the chamber via ports on the upstream and downstream faces of the chamber. The interior of the chamber was directly open to the ring vacuum. The chamber was $1.2 \mathrm{~m}$ long and had a front face that was $245 \mathrm{~mm}$ wide and $254 \mathrm{~mm}$ high. It was tapered to a width of $114.3 \mathrm{~mm}$ at the downstream end to increase the visibility of the target cell to the forward detector elements. The chamber was manufactured from a solid block of aluminum to ensure high vacuum integrity.

The upstream and downstream beamlines were made of stainless steel pipes. In order to connect the aluminum of the scattering chamber to the steel of the beam pipes, the upstream and downstream ports had Atlas 1 explosion-bonded bimetallic flanges. The aluminum sides of these flanges were welded to the scattering chamber. The steel sides were clamped to the flanges of the beam pipe, with copper gaskets in between.

\footnotetext{
${ }^{1}$ Atlas Technologies, Port Townsend, WA, USA.
}

a)

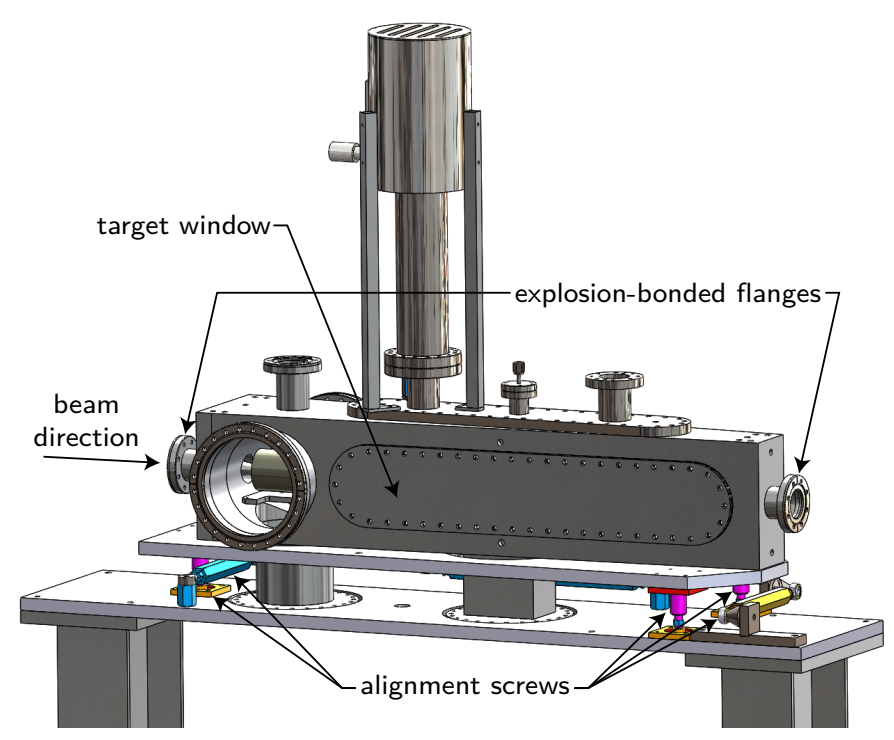

b)

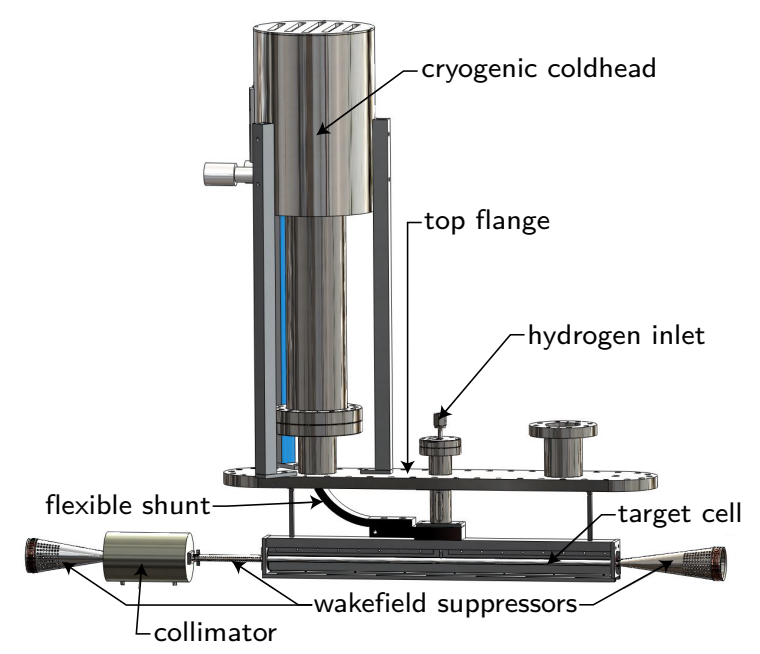

Figure 2: Design drawings of the scattering chamber (a), and the inner target system components and coldhead (b)

The top of the scattering chamber featured a long port for installing the target cell. The top flange included feedthroughs for the hydrogen gas supply, the cryogenic coldhead, and various sensors. The lateral faces of the scattering chamber had large access ports for installing the collimator as well as two long windows to allow scattered particles to exit. The components inside the scattering chamber are shown in Fig. 2 b.

The windows on each side of the chamber were made from $0.25 \mathrm{~mm}$ thick 1100 aluminum alloy foil, and were sealed using O-rings. The windows subtended a polar angle range (measured from the center of the cell relative to the beam direction) of $8^{\circ}$ to $100^{\circ}$, providing a large acceptance range for the detector. The trapezoidal shape of the chamber was chosen so that the windows extended to scattering angles below $12^{\circ}$, giving the experiment's $12^{\circ}$ luminosity monitors an unobstructed 
line-of-sight to most of the target. The trapezoidal shape also angled the windows so that leptons scattered at forward angles passed through less window material, reducing energy loss and multiple scattering.

\subsection{Target Cell}

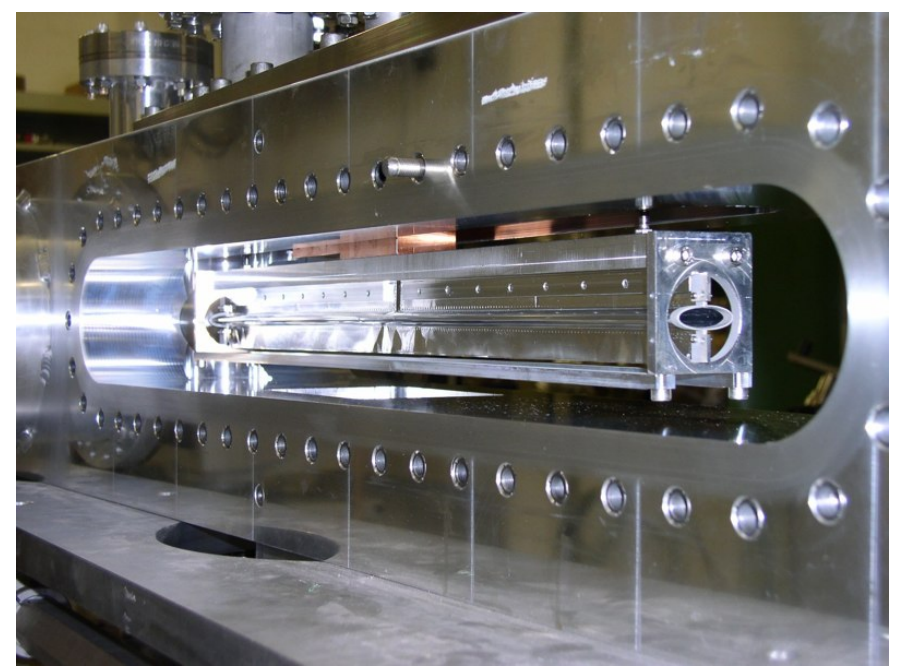

Figure 3: The target cell and frame are shown mounted in the scattering chamber, with the target windows removed.

The target cell, shown in Fig. 3, was a $600 \mathrm{~mm}$ long aluminum tube through which the DORIS beam was directed. The cell had an elliptical cross section ( $27 \mathrm{~mm}$ wide by $9 \mathrm{~mm}$ high) to match the aspect ratio of the beam envelope [5]. The tube walls were made of thin $(75 \mu \mathrm{m})$ aluminum sheets to reduce multiple scattering of outgoing particles. At the center of the tube, a small port was molded between the sheets to provide an inlet for the hydrogen gas. Once inside the cell, gas could flow only out through the ends of the tube where it was removed from the beamline by the vacuum system.

Several target cells were constructed at the Ferrara University/INFN mechanical workshop, with the experience gained from the fabrication of cells for the target of the HERMES experiment [6]. Cells were formed by the following procedure:

- Two sheets of $75 \mu \mathrm{m}$ Goodfellow ${ }^{2} 99.5 \%$ pure $\mathrm{Al}$ foil were cut, cleaned with ethanol, and then thermally softened for two hours at $270{ }^{\circ} \mathrm{C}$ between thermic glass plates to prevent foil ripples. Each sheet was $600 \mathrm{~mm}$ long and $200 \mathrm{~mm}$ wide.

- The sheets were aligned on a copper mold and formed with a pneumatic press. The foils are shown before and after the pressing process in Fig. 4 . During the process, the two halves of the gas injection tube at the center of the cell were also formed.

- After molding, the ends of each sheet were fitted with two aluminum reinforcement pieces, as shown in Fig. 5 Additionally, the reinforcements provided support for clamping the wakefield suppressors to the cell.

\footnotetext{
${ }^{2}$ Goodfellow Corporation, Coraopolis, PA, USA
}

a)

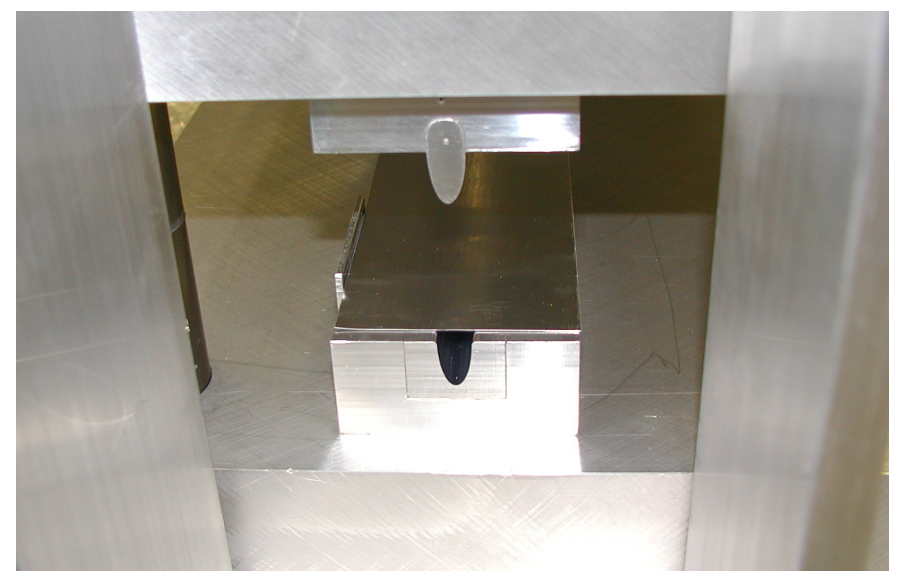

b)

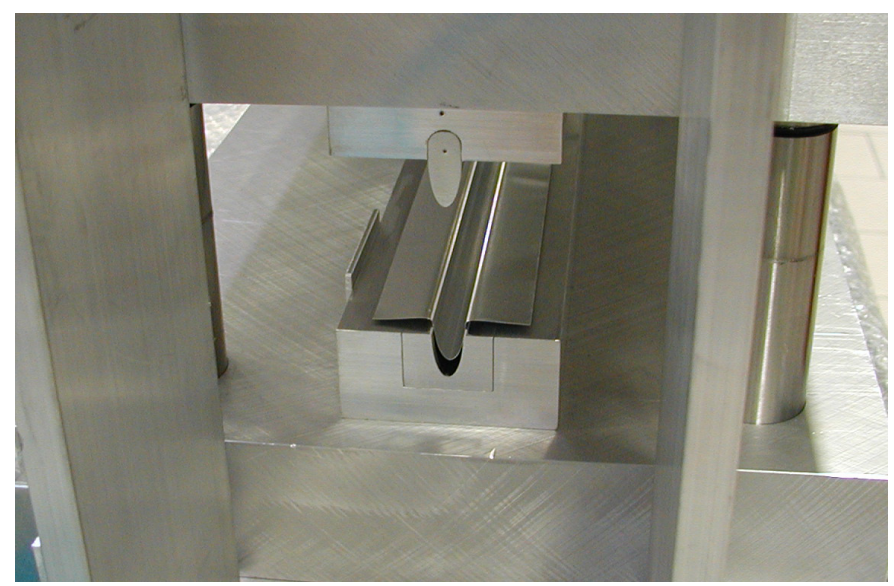

Figure 4: The target cell foil in the pneumatic press before (a) and after (b) shaping

- The two cell halves were then placed on a copper mold and spot welded together along the top and bottom seams. The mold served as one of the electrodes for the spot welding.

After a cell was fabricated, it was fixed into a mounting assembly. First, the cell was secured in an aluminum frame made of the high-purity 6063 aluminum alloy, which has excellent thermal conductivity at cryogenic temperatures. The frame provided the rigid support for a wrapping of ten layers of aluminized Mylar reflective insulation. A copper block, which served as a high-conductivity thermal junction, was attached to the top of the frame at the midpoint of the cell. The cell assembly was suspended from the top flange by a steel tube. Pins at each end of the top flange prevented the ends of the cell assembly from bending upward while under thermal stress.

The cell's position in the scattering chamber was adjusted by adding shims into the mounting assembly. Final adjustments of the cell's position were made by turning alignment screws on the scattering chamber, which moved the entire chamber relative to the beam. 


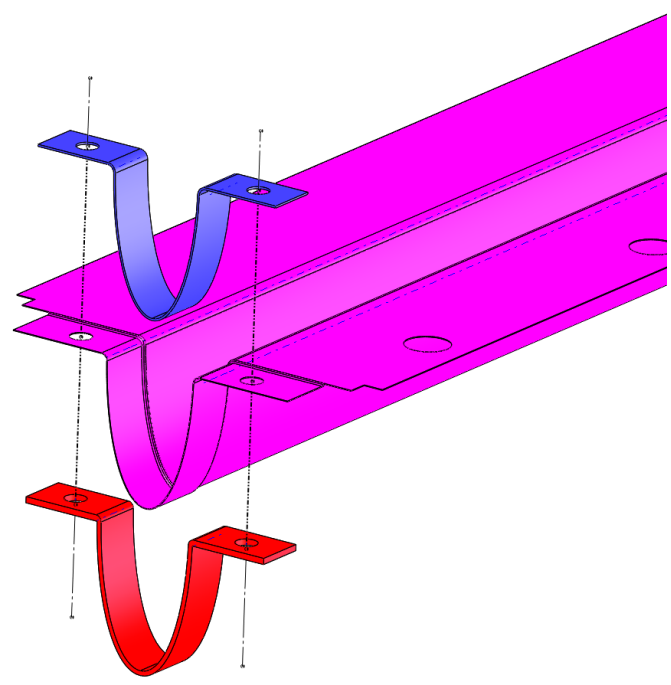

Figure 5: Schematic of the reinforcement pieces at the ends of the cell

\subsection{Collimator}

A cylindrical tungsten collimator, placed directly in front of the target cell, shielded the cell from synchrotron radiation and from the beam halo. It was fabricated from a solid cylinder of tungsten using wire electrical discharge machining. The collimator had the smallest aperture in the DORIS ring. The opening in the upstream face was elliptical, $25 \mathrm{~mm}$ wide by $7 \mathrm{~mm}$ high (approximately the $10 \sigma$ beam diameter at that location for a $4.5 \mathrm{GeV}$ beam [5]). The aperture flared towards the downstream end, to $27 \mathrm{~mm}$ wide by $9 \mathrm{~mm}$ high, to prevent smallangle scattering of synchrotron photons off the interior surface of the collimator onto the cell walls.

The outer dimensions of the collimator were selected after taking into account the results of a Monte Carlo simulation, in which electrons and positrons from the beam halo were made to impinge against the upstream face of the collimator. The particles from the resulting shower that managed to exit the collimator and the scattering chamber were counted. After varying the collimator's dimensions in the simulation, an outer diameter of $82.55 \mathrm{~mm}$ and a length of $139.7 \mathrm{~mm}$ were selected, balancing weight and cost with shielding performance. The results of the simulation are shown in Fig. 6 .

The heat load on the collimator due to incident synchrotron radiation was estimated to be on the order of $25 \mathrm{~W}$ during $4.5 \mathrm{GeV}$ running, which would easily be dissipated through the collimator supports. During $2 \mathrm{GeV}$ running, the power would be on the order of $1 \mathrm{~W}$.

\subsection{Wakefield Suppressors}

Wakefield suppressors were necessary to maintain the target cell at cryogenic temperatures by preventing heating caused by beam wakefields. The wakefield suppressors spanned gaps between conducting structures surrounding the beam to ensure continuous conductivity. Any sharp transitions or gaps in conductivity would act as electromagnetic cavities that would be

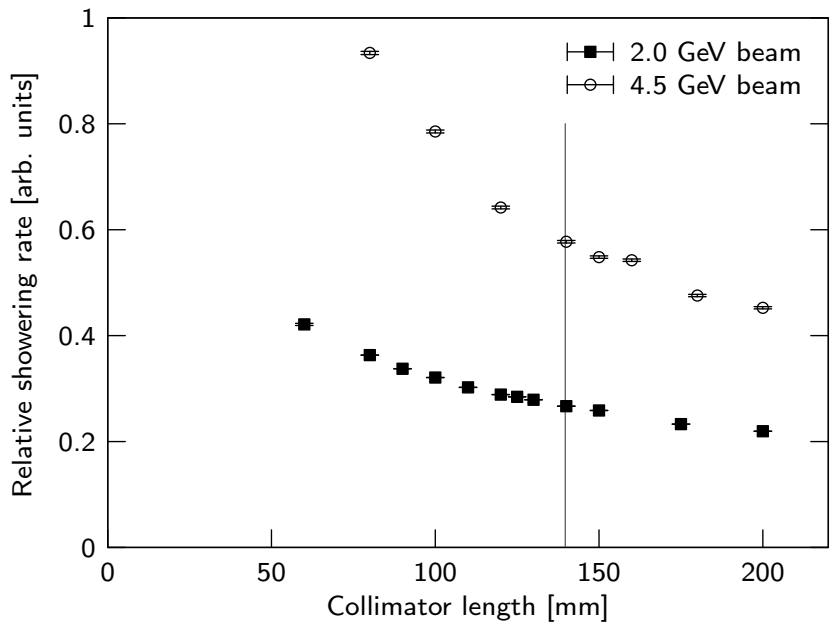

(Outer diameter fixed at $150 \mathrm{~mm}$ )

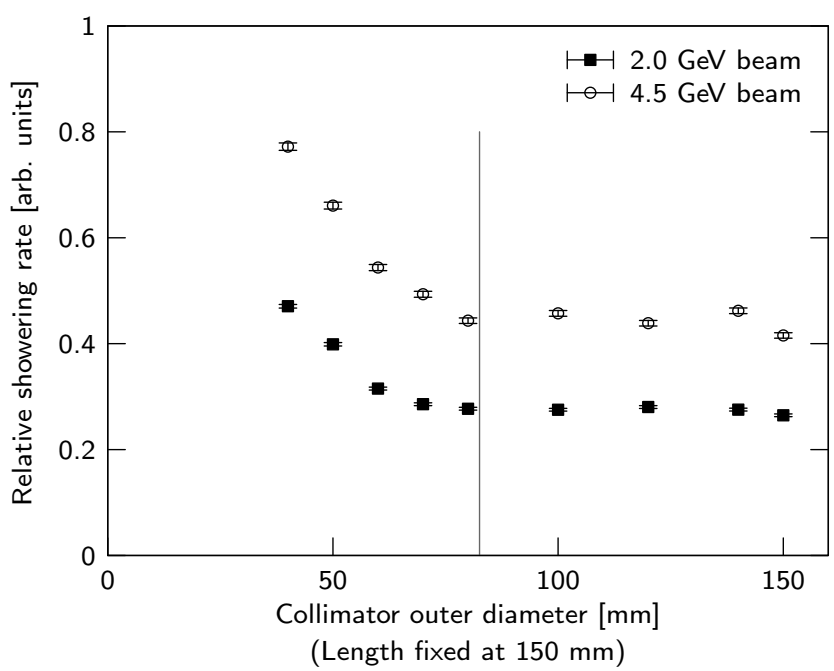

Figure 6: A Monte Carlo simulation was used to study how the dimensions of the collimator affected the showering rate from beam halo particles. A length of $139.7 \mathrm{~mm}$ and an outer diameter of $82.55 \mathrm{~mm}$ were chosen for the final design.

excited by the passing beam, causing heating in the surrounding elements. To prevent this, three wakefield suppressors were produced to cover the following transitions:

1. From the upstream $60 \mathrm{~mm}$ diameter circular scattering chamber port to the $25 \mathrm{~mm}$ by $7 \mathrm{~mm}$ elliptical opening of the collimator,

2. From the exit of the collimator to the entrance of the target cell (both $27 \mathrm{~mm}$ by $9 \mathrm{~mm}$ elliptical), and

3. From the $27 \mathrm{~mm}$ by $9 \mathrm{~mm}$ elliptical exit of the target cell to the $60 \mathrm{~mm}$ circular diameter of the downstream scattering chamber port.

The design goal was to limit wakefield heating to $10 \mathrm{~W}$.

The upstream wakefield suppressor was screwed to the upstream face of the collimator. A beryllium-copper $(\mathrm{BeCu})$ spring cone made a sliding connection with the inside of the upstream chamber port to provide good electrical contact despite thermal expansion and contraction. Since the wakefield suppressor was unshielded by the collimator and needed to be 
tolerant to synchrotron radiation and beam halo particles, it was fabricated from aluminum. Aluminum's high thermal conductivity helped to dissipate heat from radiation, while its low density offered a comparatively small cross section for beam halo particles. The wakefield suppressor had a thin coating of silver to increase its electrical conductivity.

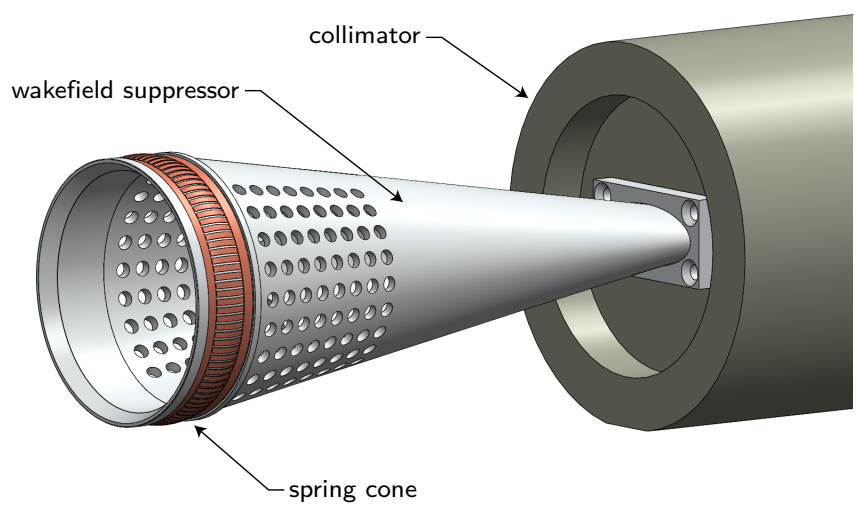

Figure 7: Design drawing of the upstream wakefield suppressor, which bridged the gap between the upstream beam pipe and the collimator

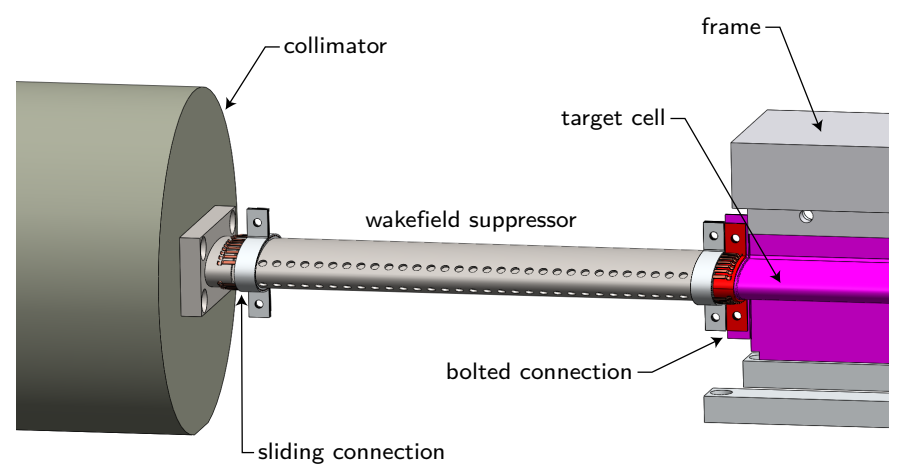

Figure 8: Design drawing of the middle wakefield suppressor, which bridged the gap between the collimator and the target cell

The middle and downstream wakefield suppressors, shown in Figs. 8 and 9, were made from silver-coated stainless steel. The middle wakefield suppressor was fixed with a bolted connection to the reinforcement piece at the upstream end of the target cell. $\mathrm{BeCu}$ spring fingers extended across the connection to maintain good electrical conductivity, even when thermal contraction produced a gap. The upstream end also had $\mathrm{BeCu}$ spring fingers, which made a sliding connection to an elliptical spout that was screwed to the downstream face of the collimator. The downstream wakefield suppressor was fixed with a bolted connection to the reinforcement piece at the downstream end of the target cell. BeCu spring fingers were used to maintain electrical conductivity across the connection. The wakefield suppressor's downstream end had a BeCu spring cone, which made a sliding connection to the downstream chamber port.

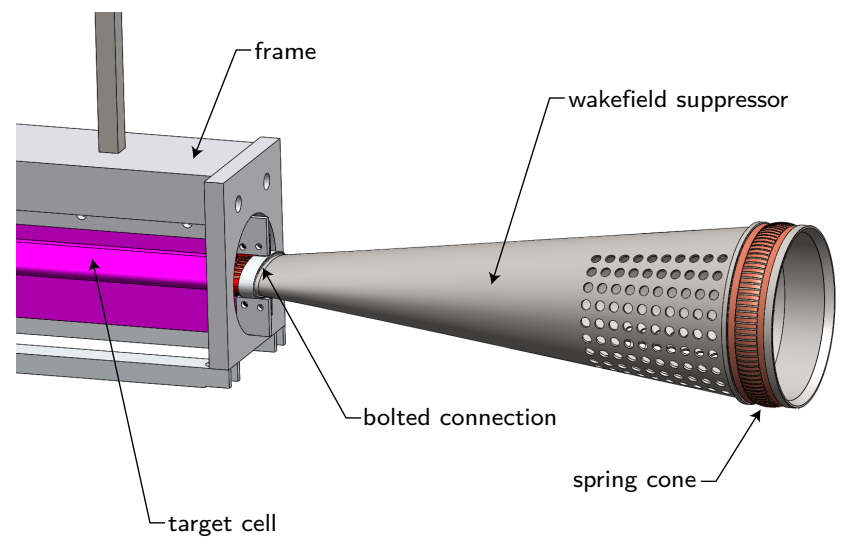

Figure 9: Design drawing of the downstream wakefield suppressor, which bridged the gap between the target cell and the downstream beam pipe

The wakefield suppressors were machined to include numerous small holes through which the target gas could escape into the scattering chamber. The locations of the holes were chosen to be as far as possible from the beam to reduce wakefield heating from the holes.

\subsection{Cryogenic System}

A cryogenic system was used to remove heat from the cell and to increased the target's density by reducing the cell's gas conductance. Since the hydrogen gas entered the cell in the molecular flow regime, it reached equilibrium temperature with the cell within a few collisions with the cell walls. Geometric calculations of the conductance of the cell suggested that a target temperature of $75 \mathrm{~K}$ would be needed to attain a target thickness of $3 \times 10^{15}$ atoms $/ \mathrm{cm}^{2}$ at a flow rate of $0.6 \mathrm{sccm}$ into the cell.

The cell was cooled by a CryoMech ${ }^{3}$ AL230 coldhead with a CP950 compressor. The coldhead was mounted directly above the scattering chamber so that it could be close to the cell but out of the way of the OLYMPUS detectors positioned in the horizontal plane. In this position, the coldhead was in a region of high magnetic field, so a cylindrical steel covering was placed over the coldhead to provide magnetic shielding. The compressor was located in the pit below the experiment and did not require magnetic shielding.

The coldhead's heat exchanger was coupled to the copper block on the cell assembly by a flexible laminated copper shunt, manufactured by Watteredge The shunt's flexibility accommodated the expansion and contraction of the target during changes in temperature and dampened mechanical vibration from the coldhead. A thin layer of indium was placed between all of the thermal couplings to improve heat conductivity.

The scattering chamber remained at room temperature and needed to be thermally insulated from the cell. The cell assembly was suspended from the chamber by a steel tube, which was fixed to the copper block by four small screws. The tube

\footnotetext{
${ }^{3}$ CryoMech, Inc., Syracuse, NY, USA

${ }^{4}$ Watteredge, Inc., Avon Lake, OH, USA
} 
was thin, to reduce its thermal conductivity. Sheets of Kapton were placed between the tube and the copper block to provide insulation.

The coldhead was rated to dissipate $36 \mathrm{~W}$ of thermal power at a temperature of $25 \mathrm{~K}$. A simulation of the thermal conductance of the target components, assuming $10 \mathrm{~W}$ of thermal power deposited on the cell, indicated that a target temperature of $75 \mathrm{~K}$ could be sustained. The results of this calculation are shown in Fig. 10.

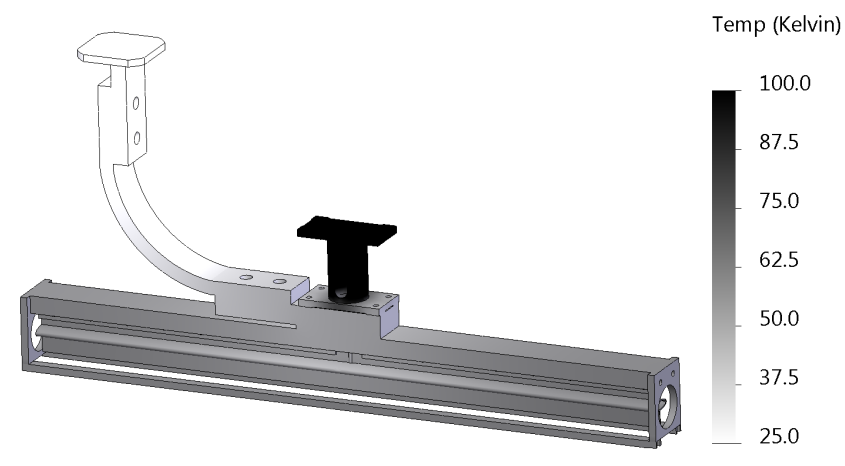

Figure 10: The temperature profile of the target cell was simulated assuming a heat load of $10 \mathrm{~W}$ on the cell. The simulation suggested that the coldhead could maintain a target temperature of $75 \mathrm{~K}$.

The temperature of the target cell was monitored by seven Pt100 temperature sensors. The sensors were positioned along the length of the cell so that the temperature uniformity could be monitored. The wires for the sensors were fed through the ports in the top of the scattering chamber.

\subsection{Vacuum System}

A system of vacuum pumps removed the hydrogen from the scattering chamber and beamline after it exited the target cell. Since the cell was internal to the beamline, the vacuum system was critical to the operation of the the target as it prevented the hydrogen from the target from spoiling the vacuum of the DORIS ring. The system included six turbomolecular vacuum pumps (Osake $2^{5}$ TG 1100M and Edwards STP 1003C), each with $800 \mathrm{~L} / \mathrm{s}$ capacity for hydrogen, and four Non-Evaporable Getters (NEGs) (SAES7 CapaciTorr CFF 4H0402), each with $400 \mathrm{~L} / \mathrm{s}$ equivalent capacity for hydrogen. Fig. 11 shows the placement of the pumps in relation to the beamline.

The vacuum system design was based on a detailed conductance map of all the vacuum components around the OLYMPUS section of the beamline. The pumps were arranged in three stages, with each stage reducing the pressure by an order of magnitude, from $10^{-6}$ Torr in the scattering chamber to $10^{-9}$ Torr in the DORIS ring. The first stage pumps (3 and 4), were positioned below the scattering chamber, the second stage pumps (2 and 5) were positioned upstream and downstream of

\footnotetext{
${ }^{5}$ Osaka Vacuum Ltd., Osaka, Japan.

${ }^{6}$ Edwards, Crawley, UK.

${ }^{7}$ SAES Group, Lainate, Italy.
}

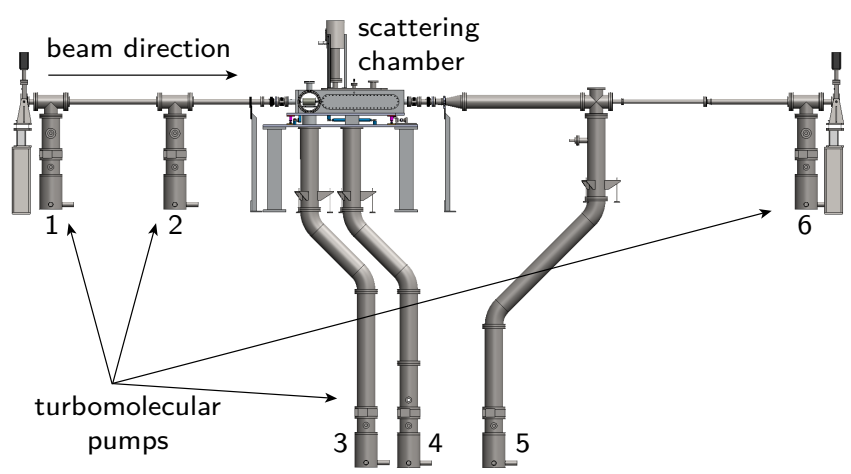

Figure 11: The vacuum system as oriented in the DORIS beamline.

the scattering chamber, while the third stage pumps (1 and 6) were positioned at the beginning and end of the OLYMPUS section of the beamline. NEGs were placed above the outer four pumps $(1,2,5$, and 6$)$ to assist in removing hydrogen at low pressure.

Since all of the turbomolecular pumps had magnetically levitating rotors, they could not operate in a magnetic field. Pumps 3,4 , and 5 were placed in the pit, several meters below the beamline, outside the fringe field of the OLYMPUS toroid magnet. To compensate for the additional pumping distance, $20 \mathrm{~cm}$ diameter pipes were used to connect the pumps to the beamline with sufficient conductance.

\subsection{Gas Feed System and Supply}

The target system employed a Parkel ${ }^{8}$ 75-34 hydrogen gas generator, which produced ultra-pure molecular hydrogen (fewer than $200 \mathrm{ppb}$ of impurities) via electrolytic dissociation of water. To create the ultra-pure hydrogen, the generator incorporated a cathode consisting of palladium tubes, into which only $\mathrm{H}^{+}$ions could pass. The ions combined to form $\mathrm{H}_{2}$ in these tubes before exiting the generator to the supply line. Only water deionized to a resistivity of greater than $5 \mathrm{M} \Omega-\mathrm{cm}$ was used in the system to ensure purity and to protect the palladium cathode. The generator operated continuously throughout OLYMPUS data taking to maintain a pressure of 20 psi on the supply line, so as to keep the line at positive pressure relative to the atmosphere and to provide an ample supply to the gas feed system.

The flow of gas into the target cell was controlled by a remotely-operated system of mass flow controllers (MFCs) and pneumatic solenoid valves, modeled on the system used for the BLAST experiment's target [7] at the MIT Bates Linear Accelerator. The MFCs provided reliable flow control in the range from approximately 0.1 to $1 \mathrm{sccm}$. Two "buffer/reservoir" tanks of precisely-known volume were attached to the system to provide a means of calibrating the MFC output. Additionally, the volumes could be used to drive the output flow using the differential pressure between the volumes, which allowed more precise control of lower flow rates. The valves and MFCs

\footnotetext{
${ }^{8}$ Parker-Hannifin Corporation, Haverhill, MA, USA
} 
were controlled during experimental running via the slow control system described in Section 2.8, but were also controllable using a console system beneath the OLYMPUS detector.

\subsection{Slow Control System}

The target slow control system used VME-based CPUs and modules. Standard VME I/O modules were used to control the valves of the gas feed system (using custom made valve control boxes) and to read the status of the turbo pump controllers. VME ADC cards were used to read the output of the pressure sensors in the target supply system and the flows measured by the MFCs. The MFC flow was set by VME DAC cards. The pressure sensor modules of the target system were equipped with RS-232 serial interfaces, which were connected to the VME CPU via off-the-shelf RS-232 to USB converters.

The software of the slow control system was divided into three parts: the interface to the hardware, a database, and the user interface. The interface to hardware was realized using the Experimental Physics and Industrial Control System $(\text { EPICS })^{9}$ software package, which also maintained an image of the current system state. The system state was replicated in a SQL database, which also recorded the history of the various data channels. The database backend was realized using PostgreSQL. The main DAQ system of OLYMPUS queried the database at constant time intervals and updated the experiment data files with information from the slow control channels. Custom interconnection daemons connected the database to EPICS and the slow control system of DESY, called TINE ${ }^{10}$. The custom graphical user interface was built on a web-based platform. A server, written in Python using the FLASK framework, provided an HTML- and JavaScript-based GUI, which was accessible from any computer with a web browser inside the DESY network. View-only access from outside DESY was realized with a replicated database and a read-only version of the server running on an exposed host, a virtual machine provided by the DESY IT department.

The complete slow control design, originally developed for the target system alone, was later adopted and extended to control all aspects of the OLYMPUS experiment (see [1]).

\section{Operations}

A prototype of the OLYMPUS target system was installed in the DORIS ring in January 2011 for a test experiment. At the end of this test, the prototype target was uninstalled and improvements were made based on the experience gained. The full target system was installed in July 2011, along with the OLYMPUS spectrometer. Commissioning tests took place during the fall of 2011. The first OLYMPUS data run took place during February 2012. The second OLYMPUS data run took place from October 2012 to the beginning of January 2013.

\footnotetext{
${ }^{9}$ Experimental Physics and Industrial Control System, Argonne National Laboratory, USA, http://www . aps . anl.gov/epics/index.php

${ }^{10}$ Three-fold Integrated Networking Environment, DESY, Germany, http: //tine.desy.de
}

\subsection{Test Experiment}

A test experiment was conducted with a prototype target system in January and February 2011. Since the OLYMPUS detector was not yet assembled, some simple detectors were arranged to detect leptons and protons in coincidence from elastic scattering in the target: a telescope of multi-wire proportional chambers and a calorimeter of lead-glass crystals were used to detect scattered leptons at $12^{\circ}$, and an array of three time-of-flight scintillator bars detected recoiling protons at back angles. During these tests, the cryogenic and vacuum systems performed well under OLYMPUS-like running conditions. Reconstruction of the scattering vertices showed that the target had the expected triangular density profile.

\subsection{Synchrotron Operation}

After the test experiment, DORIS resumed synchrotron operation. The prototype target was not able to withstand the conditions of synchrotron operation, became damaged, and had to be removed. Several flaws were identified and corrected in the final design, which was described in Section 2.4. The prototype target cell frame, which was not built from 6063 aluminum, began to warp under thermal stress. The prototype wakefield suppressors had gaps of approximately $1 \mathrm{~mm}$ at each junction, which caused excessive heating. These gaps were eliminated in the final design by the system of bolted and sliding connections. The laminated copper shunt replaced a simple copper braid to improve the heat conductivity between the target cell frame and the coldhead.

The improved target system was installed in July 2011. It performed well during OLYMPUS commissioning tests and withstood synchrotron operation, during which the target cell temperature was approximately $50 \mathrm{~K}$. For comparison, the target achieved a temperature of approximately $40 \mathrm{~K}$ with no beam.

\subsection{OLYMPUS Run I}

During the first OLYMPUS run, data were collected with beam currents between 40 and $65 \mathrm{~mA}$, divided in ten bunches. The target was operated with a flow of $0.8 \mathrm{sccm}$ while maintaining a satisfactory beam lifetime on the order of 30 minutes. In these conditions, the target had a temperature of approximately $55 \mathrm{~K}$.

Analysis of the luminosity monitor data from the first run indicated that the luminosity had only been one eighth of what was expected from the set flow rate. Inspection revealed a leak in the junction between the tube that delivered the hydrogen gas to the cell assembly and the cell itself. Most of the hydrogen was leaking into the scattering chamber without ever entering the target cell. It was not possible to fix the leak in situ, so the scattering chamber was removed. A new cell was installed, with a bayonet to bridge the gap between the two tube pieces. This modification prevented any leak at this junction. 


\subsection{OLYMPUS Run II}

The elimination of the leak increased the target thickness to the design value, resulting in a significant reduction of the DORIS beam lifetime compared to Run I. The short lifetime was overcome by operating in "top-up" mode. In this mode, the storage ring was refilled to the desired current every few minutes rather than allowing the beam current to decay significantly. With $0.6 \mathrm{sccm}$ flow, a typical beam lifetime was on the order of only ten minutes, but in top-up mode, an average beam current of more than $60 \mathrm{~mA}$ was sustained during data taking. At that flow, the pressure gauges at the central pumps reached a pressure of $2 \times 10^{-6}$ Torr. The upstream pumps recorded pressures of approximately $1 \times 10^{-7}$ and $2 \times 10^{-8}$ Torr. The downstream pumps recorded pressures of $5 \times 10^{-7}$ and $3 \times 10^{-8}$ Torr. Gauges just outside the OLYMPUS beamline segment recorded pressures of $7 \times 10^{-8}$ Torr and $2 \times 10^{-8}$ Torr. The average pressure in the DORIS ring was maintained at $3 \times 10^{-9}$ Torr.

During the second run, a target temperature slightly below $70 \mathrm{~K}$ was maintained, as shown in Fig. 12. Synchrotron operation was expected to produce more severe wakefields because of the higher currents and fewer bunches, but this turned out not to be the case. The higher temperature during OLYMPUS running was likely caused by the target enlarging the beam and increasing the wakefield heating.

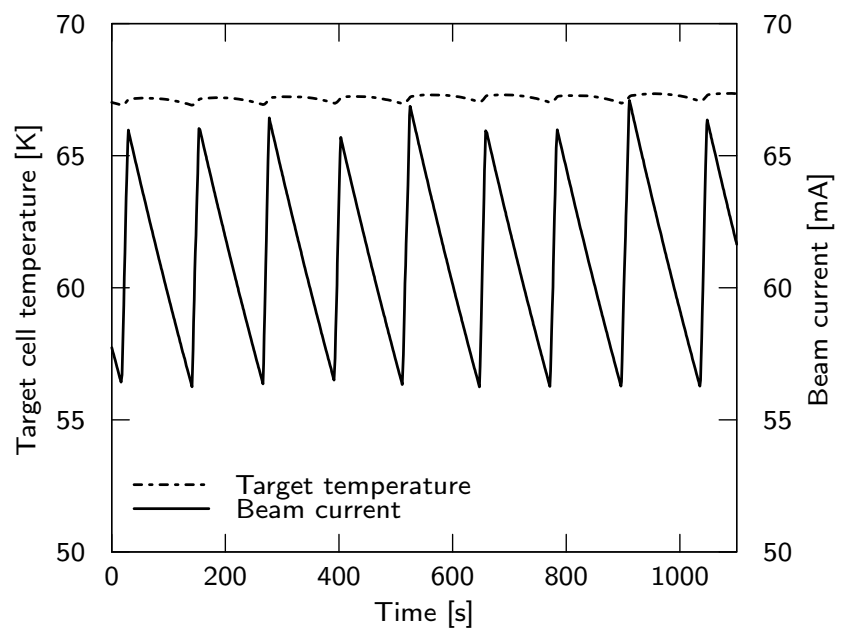

Figure 12: During typical running, the target cell temperature was stably maintained in top-up mode, with only a small dependence on the beam current.

In a preliminary analysis, it was possible to reconstruct the target density distribution from a small subset of the full data set. The result is shown in Fig. 13. The distribution mainly follows the expected triangular shape but is slightly shifted to negative positions, i.e. upstream. The distribution is also slightly wider than the cell. Further analysis will examine whether this is real or an effect of the preliminary tracking and event selection.

\section{Conclusion}

The design and realization of the OLYMPUS target system has been presented. The OLYMPUS experiment required a

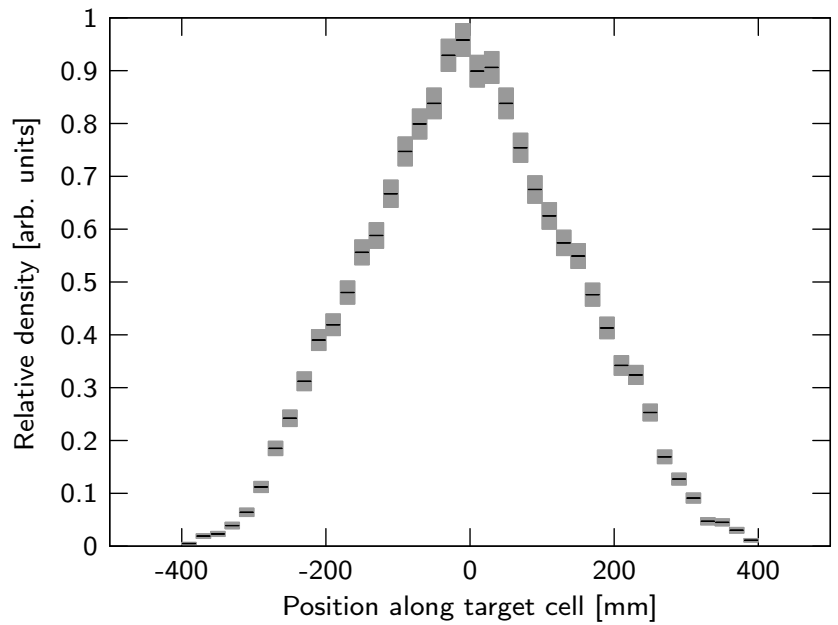

Figure 13: The reconstructed target density distribution follows the expected triangular shape. An offset to negative positions and tails beyond the expected range of $\pm 300 \mathrm{~mm}$ are visible.

unpolarized hydrogen gas target that could withstand a harsh beam environment. The target used an internal gaseous design to avoid degrading the DORIS beam. The cell was cryogenically cooled to increase the target thickness for a given flow. With significant wakefield suppression, the target performed to specifications, and a temperature of under $70 \mathrm{~K}$ was sustained during operation. The analysis of data taken during the OLYMPUS experiment is ongoing.

\section{Acknowledgements}

We would like to thank Chris Vidal as well as the MIT-Bates technicians for helping to realize and install the OLYMPUS target. Additionally, we thank the members of the DORIS vacuum group for their assistance. We gratefully acknowledge the input from Rainer Wanzenberg and Uli Kötz, whose correspondence regarding wakefields and beam halo were instrumental in the design of the target and collimator. We thank Uwe Schneekloth for coordinating the various efforts at DESY and for his helpful suggestions during the course of the target design and modification. We gratefully thank our other collaborators in the OLYMPUS experiment, who made the successful completion of the experiment possible and collected the data used to create Fig. 13 We especially would like to express our gratitude to Alexander Winnebeck and Jürgen Diefenbach who lent us invaluable assistance on numerous occasions.

This work was supported by the Office of Nuclear Physics of the U.S. Department of Energy.

\section{References}

[1] R. Milner, D. Hasell, M. Kohl, U. Schneekloth, N. Akopov, et al., The OLYMPUS Experiment, Nuclear Instruments and Methods in Physics Research Section A: Accelerators, Spectrometers, Detectors and Associated Equipment 741 (2014) 1-17.

[2] P. A. Guichon, M. Vanderhaeghen, How to reconcile the Rosenbluth and the polarization transfer method in the measurement of the proton formfactors, Phys. Rev. Lett. 91 (2003) 142303. 
[3] J. Arrington, P. Blunden, W. Melnitchouk, Review of two-photon exchange in electron scattering, Prog.Part.Nucl.Phys. 66 (2011) 782-833.

[4] D. Hasell, T. Akdogan, R. Alarcon, W. Bertozzi, E. Booth, et al., The BLAST experiment, Nucl.Instrum.Meth. A603 (2009) 247-262.

[5] F. Brinker, private communication, 2009.

[6] A. Airapetian, et al., The HERMES polarized hydrogen and deuterium gas target in the HERA electron storage ring, Nucl.Instrum.Meth. A540 (2005) 68-101.

[7] D. Cheever, E. Ihloff, J. Kelsey, H. Kolster, N. Meitanis, et al., A highly polarized hydrogen/deuterium internal gas target embedded in a toroidal magnetic spectrometer, Nucl.Instrum.Meth. A556 (2006) 410-420. 